Cite this: Phys. Chem. Chem. Phys., 2014, 16, 6859

Received 9th December 2013, Accepted 17th February 2014

DOI: $10.1039 / c 3 c p 55185 \mathrm{~g}$

www.rsc.org/pccp

\title{
A size resolved investigation of large water clusters
}

\author{
Udo Buck, ${ }^{a}$ Christoph C. Pradzynski, ${ }^{\text {b }}$ Thomas Zeuch, ${ }^{b}$ Johannes M. Dieterich ${ }^{c}$ and \\ Bernd Hartke ${ }^{c}$
}

\begin{abstract}
Size selected water clusters are generated by photoionizing sodium doped clusters close to the ionization threshold. This procedure is free of fragmentation. Upon infrared excitation, size- and isomerspecific $\mathrm{OH}$-stretch spectra are obtained over a large range of cluster sizes. In one application of this method the infrared spectra of single water cluster sizes are investigated. A comparison with calculations, based on structures optimized by genetic algorithms, has been made to tentatively derive cluster structures which reproduce the experimental spectra. We identified a single all-surface structure for $n=25$ and mixtures with one or two interior molecules for $n=24$ and 32. In another application the sizes are determined at which the crystallization sets in. Surprisingly, this process strongly depends on the cluster temperature. The crystallization starts at sizes below $n=200$ at higher temperatures and the onset is shifted to sizes above $n=400$ at lower temperatures.
\end{abstract}

\section{Introduction}

Water is one of the most widely spread substances on earth. It is essential for life and it plays, in addition, a crucial role in chemical, biological, atmospherical, and interstellar systems. That is also valid for water clusters. Typical examples are the growth and nucleation of water and water containing nanoparticles and the chemical reactions at their surfaces. ${ }^{1-5}$ The special properties of water are dominated by the hydrogen-bonded (HB) network. Thus the unusual differences in the density of liquid and solid water are traced back to the arrangement of the HB-network. In ice it consists of six-membered rings of exactly four-coordinated water molecules in a well-defined threedimensional array. In the case of liquid water in addition fivemembered rings as well as three-coordinated molecules appear and the complete arrangement also fluctuates on a ps-time scale so that direct measurements are difficult to perform. ${ }^{6,7}$ Water clusters of finite size are to a certain extent an ideal tool to understand these different macroscopic properties on a microscopic level and to answer fundamental questions of the kind as follows. At what cluster size typical structures of the bulk like completely four-coordinated molecules appear? In which size region the transition to completely crystalline behaviour

\footnotetext{
${ }^{a}$ Max-Planck-Institut für Dynamik und Selbstorganisation, Am Faßberg 17, 37077 Göttingen, Germany. E-mail: ubuck@gwdg.de

${ }^{b}$ Georg-August-Universität Göttingen, Institut für Physikalische Chemie, Tammannstraße 6, 37077 Göttingen, Germany. E-mail: tzeuch1@gwdg.de ${ }^{c}$ Theoretische Chemie, Institut für Physikalische Chemie, Universität Kiel, Olshausenstr. 40, 24098 Kiel, Germany.E-mail: hartke@pctc.uni-kiel.de
}

does occur? To answer such questions a sensitive experimental method is necessary. A quite general one is the infra-red (IR) spectroscopy of the OH-stretch motion which directly probes the $\mathrm{H}$-bond between $\mathrm{H} \cdots \mathrm{O} .{ }^{8-11}$ Very recently, the first Raman scattering spectra of the $\mathrm{OH}$ stretching mode in small, isolated water clusters have also been reported. ${ }^{12}$ Because of the large red-shift of more than $700 \mathrm{~cm}^{-1}$ from the free $\mathrm{OH}$ to a strongly $\mathrm{H}$-bonded $\mathrm{OH}$, a method very sensitive to the strength of the HB-network is created. The crucial condition for the application of this method to larger clusters is the size selection. ${ }^{8,11}$ Clusters are generally produced with a size distribution and typically detected and size selected by mass spectrometry. However, in the ionization process they usually fragment. Thus special methods are required to circumvent this problem.

The most general one is the deflection of clusters in a scattering experiment with an atomic beam. By specifying the angle and the velocity after the collision the size can finally be resolved independent of the detection method. ${ }^{13}$ Vibrational predissociation is then applied to obtain the IR spectrum of the size selected cluster. But this method has only proven to be applicable to sizes up to $n=12 .^{8,14,15}$ Similar results in this size range have been obtained by other methods based on rotational resolution, ${ }^{16}$ argon tagging, ${ }^{17}$ or the addition of aromatic molecules and their detection by UV-excitation. ${ }^{18-20}$ The latter method has also been applied to water cluster sizes up to $n=50$ by adding phenol. ${ }^{11,21,22}$ But the size can only be fixed close to $n+\Delta n$ with $\Delta n$ between 0 and 6 , because of possible fragmentation processes in the detection after an unspecific UV-excitation.

A viable method to solve this problem is to dope the water clusters with a single Na atom. The low ionization energy of 
$5.14 \mathrm{eV}$ and the easily available tunable UV lasers in this energy range allow us to perform a fragmentation-free detection by the photoionization at the threshold. In this way the size distributions of ammonia, water, and argon clusters have been measured. ${ }^{23,24}$ The OH-stretch spectra using the Na doping method have been obtained as follows. The UV-laser energy is tuned to the appearance ionization energy, which is a rather broad region around $3.1 \mathrm{eV}$. IR excitation of the clusters before photoionization leads to an enhancement signal, which is used to generate the desired spectrum. ${ }^{25}$ The resulting spectra, obtained in the first application of this method, were those of $\mathrm{Na}\left(\mathrm{H}_{2} \mathrm{O}\right)_{n}$ and for smaller clusters they were clearly dominated by features of the solvated electron. ${ }^{25,26}$ In the meantime we found out that for many experimental conditions the IR spectra are even for smaller clusters dominated by a class of isomers, in which the $\mathrm{Na}$ is attached to the intact water or methanol cluster. ${ }^{27-29}$ For larger clusters the influence of the single $\mathrm{Na}$ atom anyway disappears and essentially the spectra of the pure water clusters are recorded. This approach is the basis for obtaining size resolved IR spectra over a broad range of water cluster sizes, which will be presented in this perspective article.

We will start with a detailed description of the new experimental method. Then an overview of the theoretical aspects of the determination of the minimum energy configuration of the different cluster sizes and of the calculations of the corresponding $\mathrm{OH}$-stretch spectra will be given for pure water $\left(\mathrm{H}_{2} \mathrm{O}\right)_{n}$ clusters. After these foundations in the experimental and theoretical treatment special examples will be discussed. We start with $n=25$ for which the experimental data could be well reproduced by an all-surface structure. For $n=24$ and $n=32$ mixtures which also contain one or two interior water molecules were found to describe the experimental results. We close with revisiting the results for $n=6$, the smallest water cluster size with three dimensional HB networks as minimum energy configurations. This size has attracted much interest because several isomers of similar stability are present.
In the next section the focus will be directed towards much larger clusters. The experimental approach presented in this perspective article makes it possible to solve an old problem, the detection of the onset of crystallisation of $\left(\mathrm{H}_{2} \mathrm{O}\right)_{n}$ in a fully size selective experiment. It was expected from detailed calculations by Buch et al. that clusters up to $n=200$ exhibit an amorphous structure and sizes above $n=600$ show a crystalline behaviour. ${ }^{30}$ This was supported by measurements of the IR-spectra with size distributions. Similar results were obtained by electron diffraction measurements which estimate the transition between $n=200$ and $n=1000 .{ }^{31}$ In our experiments the detailed transition is measured and the dependence on the temperature is also explored. Finally, the possibilities of revealing water cluster structures, opened by the now available facilities for a combined theoretical-experimental effort, are briefly presented.

\section{Experimental methods}

The experimental set-up and the general mechanism for the modulation of the photoionization by IR excitation are illustrated in Fig. 1. In the experiment the clusters are formed in a skimmed supersonic expansion and then doped with a single sodium atom during their passage through the pick-up cell. Depending on the beam velocity (typically around $1500 \mathrm{~m} \mathrm{~s}^{-1}$ for He seeded expansions) they reach after several hundreds of microseconds the zone for interaction with IR and UV-vis radiation. A first important point here is a special property of sodium doped water (and some other hydrogen bonded) clusters. Different from the classical ammonia alkali metal case, the ionization energy evolution stops at rather small cluster sizes, for water e.g. at $n=15$. This surprising observation is a long known and intensively discussed phenomenon and closely connected to the formation of solvated electrons in clusters. ${ }^{32,33}$ Recent experimental and theoretical investigations revealed that
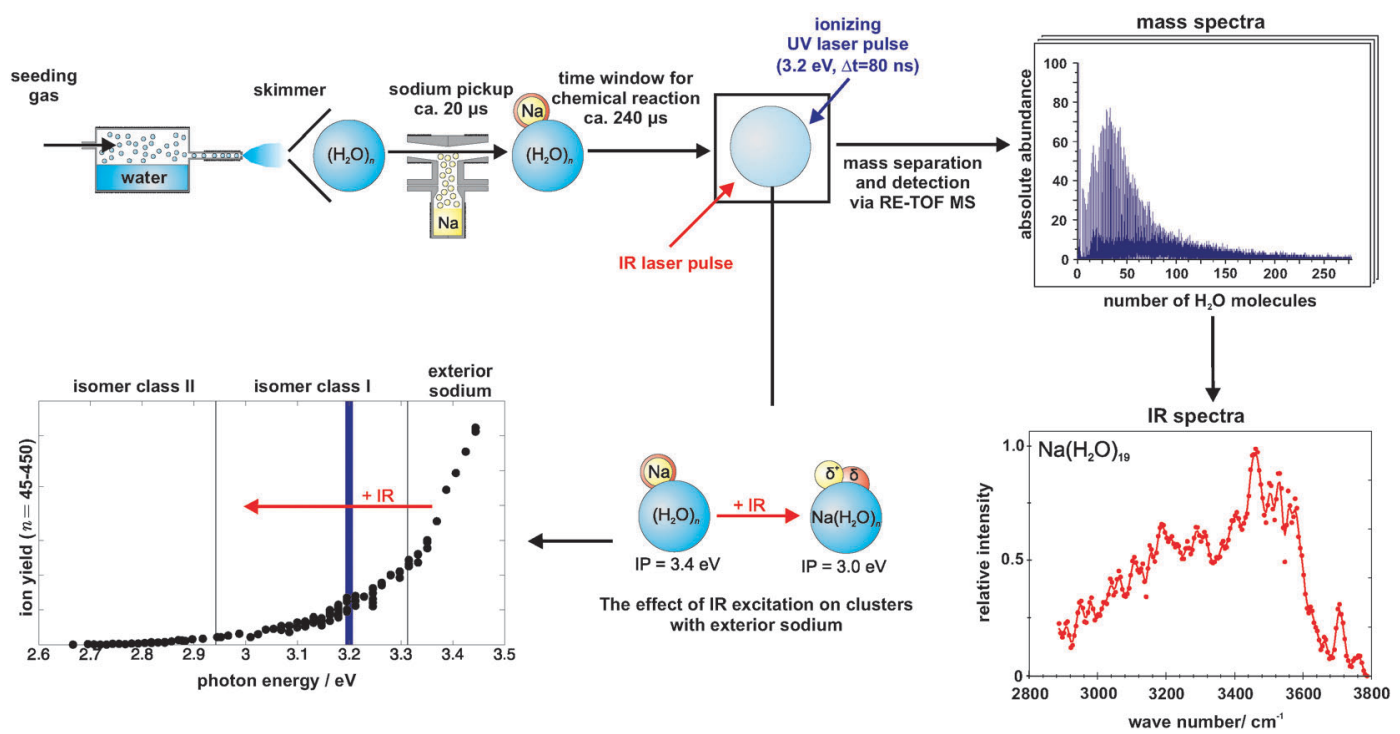

Fig. 1 Experimental configuration, photoionization scheme and origin of action effect, partly taken from ref. 29. Details are explained in the text. 
a surface electron is formed, either as contact ion pair (isomer class I) or a more loosely bound solvent separated ion pair (isomer class II). ${ }^{34}$ The ionization energy of these species is independent of the cluster size above the critical size for the emergence of isomer class II. ${ }^{34}$ As a consequence, the complete cluster size distribution (above $n=15$ ) becomes visible even when the photoionization is performed at the threshold. Therefore a single IR scan at a fixed UV/vis-frequency provides the spectrum for each size of the distribution, which is a multiplex advantage of this approach compared to size selective IR action spectroscopy with ionic clusters and the beam deflection method discussed above for neutral clusters. The second important point is the shape of the photoionization spectrum for larger clusters. Above $3.3 \mathrm{eV}$ a steep rise of ion yields with increasing photon energies is observed (lower left part of Fig. 1). At $4 \mathrm{eV}$ the ion yield is more than a factor of five higher that at $3.3 \mathrm{eV}$ (not shown). We attribute this feature to the presence of a third isomer class with higher ionization energies, in which the sodium atom is attached to the surface of the intact cluster. This second property gives rise to an ionization mechanism which allows for generating IR spectra of the undisturbed, intact water clusters. ${ }^{29}$ In our experiment the IR photons are mainly absorbed by the latter, predominant isomer class, in which the surface attached sodium atom acts as a kind of IR sensitive photopaper. Without IR excitation these clusters are invisible because of their higher IP. Upon heating by IR photon absorption a solvated electron is released from the sodium atom in the now warmer clusters. As a consequence their ionization energy has dropped below the ionizing photon energy and the clusters become visible. This mechanism works so efficiently because (a) the high abundance of clusters with exterior sodium atom maximizes IR photon absorption exploitable for the action effect, (b) the low ion signal of clusters with interior sodium atom maximizes the signal enhancement upon IR excitation and (c) the threshold photoionization minimizes the effect of cluster fragmentation. The experiment has been optimized so far for water clusters. However, the concept is general and could be adaptable for other, even very different substance classes, for example by using different metals or chromophores. The prospect of taking IR spectra over the complete cluster size distribution with single constituent resolution in a single IR laser scan seems appealing.

\section{Theoretical methods}

Totally independent of whatever factors are controlling water cluster structures in any experiment, fundamental physical laws dictate that the structures occurring predominantly will not be those with the highest possible potential energies. Hence, any theoretical examination will have to begin with finding low-energy structures. This is a difficult task already for larger clusters of isotropic particles, because the structural search space size increases exponentially with the number of particles, possibly as a general characteristic of such problems ${ }^{35}$ but certainly in practice. For non-isotropic particle interactions, an additional orientational search space, also scaling exponentially, ${ }^{36}$ is intertwined with the positional one, aggravating the scaling even further. Hence, for water clusters $\left(\mathrm{H}_{2} \mathrm{O}\right)_{n}$ of increasing sizes $n$, it becomes very quickly impossible to find the best structures with multistart local search, even if simple empirical force fields are used. Instead, starting from about $n=15$, unbiased global search has to be employed to find useful low-energy structures within practically affordable computing times.

As already mentioned in the introduction, several previous studies have established excellent agreement between experiment and theory for structures of pure, neutral water clusters up to $n=12$. Since then, a few theoretical studies have employed global optimization to larger clusters: an important benchmark was provided by Wales and Hodges ${ }^{37}$ for $\left(\mathrm{H}_{2} \mathrm{O}\right)_{n}$ up to $n=21$, employing the common TIP4P potential. More accurate model potentials from the TTM-series ${ }^{38}$ were used to predict global minimum structures ${ }^{39,40}$ up to $n=34$ and to tentatively explore the region up to $n=100$. The results up to $n=21$ were later validated $^{41}$ at the MP2 level of theory. Recently, Kazachenko and Thakkar $^{42}$ pushed the limit of serious predictions for global minima up to $n=55$, employing several TIP4P versions, AMOEBA and again a variant of the TTM2 potential. However, all these efforts so far had no direct connection to experiment.

With the new experimental developments described here, the opportunity opens up to directly compare theory and experiment beyond $n=12$.

For completeness, we briefly sum up our theoretical approaches in the following subsections. More details can be found in the literature cited in each subsection.

\subsection{Global structure optimization}

Global cluster structure optimization in general has been reviewed several times. ${ }^{43,44}$ A short but sufficiently comprehensive summary of applying such methods to water clusters can be found in the introduction of ref. 42. Hence, here we provide only the technical details on how the results presented in Section 4 were generated.

For all cluster structure predictions we have used genetic algorithms (GA) for global cluster structure optimization, as implemented in our ogolem framework. ${ }^{45}$ An important difference to standard GA implementations (which are discussed in detail in ref. 46) is the usage of a genetic pool, as opposed to a generation-based scheme. Among the advantages of using the pool scheme is the minimization of tunables and the built-in feature of elitism, thereby providing a more rapid and robust convergence towards the global minimum. Additionally, parallelization is easier and more efficient. ${ }^{40}$

As water cluster structure optimization is a challenging optimization problem even for advanced implementations, we augment our $\mathrm{N}$-species phenotype crossover operator ${ }^{47}$ with some heuristic extensions in order to achieve maximal efficiency. With a $50 \%$ probability, the cluster is rotated to its center of quality prior to the phenotype cut as discussed in ref. 48 , in order to facilitate the mating of optimal parts and the removal of cluster parts with suboptimal hydrogen bonding patterns. In order to optimize the hydrogen bonding pattern on a more 
local scale, directed mutation ${ }^{48}$ is carried out with a probability of $50 \%$ of the $5 \%$ mutation ratio instead of the standard genotype mutation on the real-number encoded coordinates.

The last heuristic Ansatz makes use of the fact that prior work $^{39,40}$ indicates the importance of two major structural groups for water clusters of this size regime, those dominated by cubic arrangements of water molecules and those dominated by other (e.g., cage-like) arrangements. As discussed in ref. 48, the percentage of near ninety-degree angles in the clusters seems to be a good measure, which we want to dub cubicity. We use this measure to construct niches to enhance the structural sampling of all groups in the genetic pool.

After assigning each solution candidate a cubicity, the algorithm attempts to update the genetic pool. In contrast to the simpler non-niching case, we do not only check if the fitness of the solution candidate is better than the least fit current entry in the pool. Instead, an additional constraint is that the pool has to remain diverse overall. For this we employ a niching strategy. Niching introduces only two new tunables, the number of niches, which essentially corresponds to an equidistant binning of the cubicity, and the maximum number of individuals allowed per niche, $N_{\text {maxpop }}$ After mapping the cubicity of the current individual to a niche, the population of this niche is assessed. If it is below the maximum population, the individual can be added to the genetic pool. If the current population matches the maximum population, the new individual will be added and the individual within this niche with the worst fitness will be reaped. This protocol enforces that at no point during the optimization more than $N_{\text {maxpop }}$ individuals in the genetic pool of size $M$ are of a certain character. If $N_{\text {maxpop }} \ll M$, maximum genetic diversity is ensured. The only potential drawback may be a slight reduction in convergence to the global minimum for simple, multimodal functions with a single funnel character (e.g. represented by Ackley's function ${ }^{49}$ ). Despite that such easy problems anyway do not pose a major challenge to our framework, ${ }^{50}$ work is underway in our lab to assess and minimize this behavior.

We want to stress that niching as a general concept is neither new nor a very complicated technology. As a feature to enhance cluster structure optimization it was implemented by one of the authors in ref. 48 , in a fashion similar to the one used here.

Specifically, we used pool sizes in the range of 100-400 and number of global optimization steps in the range of 250 000-2 500 000. The maximum number of individuals per niche was typically set to 20-25. From the palette of crossover/ mutation algorithms in ogolem, ${ }^{45}$ we employed aland2, sweden 3 and svalbard2. The precise configuration chosen depends on the nature of the optimization problem at hand. As noted previously, ${ }^{48}$ the difficulty does not increase monotonically with the cluster size.

In contrast to a common misconception, the location of the global minimum is just one important result of a global optimization run. On the way to finding the global minimum, most relevant basins of attraction in the search space are explored and partly exploited. Thereby, the final genetic population can be seen as a sampling of the most relevant minima in the conformational space. The only criterion to be met for this assertion to hold is to maintain a sufficient diversity at any point during the global optimization. As our protocol includes both an energetic diversity criterion (all minima in the pool must differ at least by $1 \times 10^{-5}$ Hartree) and a geometric diversity criterion through niching with respect to the cubicity of the solution candidates, we are confident that our protocol maintains sufficient diversity.

We want to note here that no abstract visualization of the energy landscape is obligatory for our protocol. A straightforward graph representation, ${ }^{51,52}$ now dubbed disconnectivity graphs, ${ }^{53}$ and the lid/threshold method ${ }^{52,54,55}$ will certainly prove to be a valuable extension in the future to assess how the structures in the final solution pool are connected on the energy landscape. At this point they are not required in order to draw a concise picture, though.

The potential function itself is provided by our fast Scalabased implementation of the polarizable TTM3-F force field, ${ }^{56}$ the latest in the TTMX-F series. This dedicated water force field provides accurate energetics at a reasonable computational footprint, which is of utmost importance to ensure sufficient search space coverage in any global optimization scheme. For the spectra simulations, a mix of methods was used to retain consistency with previous studies. This is discussed in detail in the next section.

\subsection{Spectra simulation}

To retain connection with previous studies, and for its superior combination of accuracy and speed, we have opted to perform IR spectra simulations with the empirical model by Victoria Buch, which was already used with great success in elucidating the experimental IR spectra for $n=7-12 .{ }^{15,57}$ Briefly, this method employs an exciton ansatz with intra- and intermolecularly coupled, anharmonic OH oscillators, based on a modified EMP potential. The frequencies of these oscillators are modified by the local electric field at the respective $\mathrm{OH}$ bond, induced by all partial charges on the surrounding molecules. Free parameters in this model, as well as this functional frequency-field dependence, were fitted by Victoria Buch to reproduce experimental IR peaks for the size range $n=7-12$. We have obtained good results with this model also for larger clusters, after very minor modifications of the frequency-field curve in the range from 3300 to $3000 \mathrm{~cm}^{-1}$. The overwhelming advantage of this model is computational speed: for any of the clusters studied here, the full spectrum is obtained in less than one second CPU time on a desktop computer. In contrast, a DFT-based spectrum takes several hours of wallclock time, on several workstation CPUs in parallel, and it produces only harmonic, uncoupled vibrations.

\subsection{Reliability considerations}

At this point, we would like to put in a word of caution, regarding the interpretation of the theoretical results presented in the following subsections: first of all, the energy difference between successive structural isomers decreases markedly with cluster size, regardless of whether these isomers are similar or totally different. Additionally, zero-point vibrational energy (ZPVE) 
and temperature (entropy) do change the preferred isomer pattern strongly. This was demonstrated long ago already for water clusters as small as $n=6 .^{58}$ The strong decrease of isomer energy differences with cluster size indicates that these effects will even be stronger for larger clusters. In a series of papers, ${ }^{59-61}$ Lenz and Ojamäe have shown these large effects, up to $n=100$.

To be more specific for the present case: in our global structure optimizations, we typically find about 30 isomers within an energy interval of $10 \mathrm{~kJ} \mathrm{~mol}^{-1}$, at the top of an energy-ordered list of the best structures. Although TTM2-F and TTM3-F have been fitted to $a b$ initio data, ${ }^{38,56}$ at these cluster sizes we do not expect an accuracy of better than $10 \mathrm{~kJ} \mathrm{~mol}^{-1}$ in total energies. However, even if these deviations were not present, the effects mentioned above (ZPVE, temperature, etc.) would be strong and hard to take into account. Actually, standard recipes to correct for these effects are bound to fail for larger water clusters. To illustrate this claim, let us quote one surprising number: in a test calculation at the BP86/6-31G(d) level, we determined the harmonic and the anharmonic ZPVE correction to a randomly selected $\left(\mathrm{H}_{2} \mathrm{O}\right)_{25}$ cluster structure. The difference between these two values amounts to $20 \mathrm{~kJ} \mathrm{~mol}^{-1}$. Hence, already the choice between doing the ZPVE correction harmonically or anharmonically will likely overturn the structural isomer ordering completely, for the best 20-40 structures. Additionally, the harmonic calculation took 17 CPU-hours (including local re-optimization of the structure at this level), the anharmonic one 984 CPU-hours (without further optimization). In short, for correcting these effects, one has the choice between feasible calculations of insufficient accuracy and more reliable calculations that just take too long. Therefore, the present aim only is to provide indications that the experimental spectra may result from a few particular isomers among the 20-40 best ones we have found. This clearly limits the conclusions that can be drawn from the results presented here. For example, at the present stage, we do not think that there is sufficient reliability in the theoretical models and data to decide questions like kinetic $v s$. thermodynamic control in the experimental cluster formation, for water clusters in the size range presented here.

Similar reliability considerations apply for the experimental results. At the moment it is not clear to what extent kinetic stabilisation may effect the isomer distribution probed by the experiment. The spectral features used to identify isomers, whose $\mathrm{OH}$ stretch spectra fit the experimental traces, are observed in several IR laser scans showing similar absorption features. However, future improvement in the experiment may allow for obtaining spectra with better quality. Therefore, the structural assignments made in the next section should be regarded as a first attempt, which is, however, for the first time based on a combined analysis of completely size selected experimental and theoretical data in this size range of water clusters.

\section{Exemplary results}

For each of the cases presented in the following $(n=24,25,32)$, we have proceeded by the following steps: several (5-10) global optimization runs at the TTM3-F level were performed, with a typical pool size of 100 . All results were collected and ranked by energy; remaining duplicates were eliminated. Then, a simple Boltzmann weighting in the experimental temperature range was used to eliminate structures with weights of less than $0.1 \%$. The remaining structures were then re-optimized with the modified EMP potential and fed into IR spectra simulation with the Buch method. By visual inspection, isomers were eliminated that exhibited spectral features grossly deviating from the experimental pattern. If more than one final structure remained, an attempt was made to produce a weighted average spectrum of them by hand. This procedure should be understood as a proofof-principle approach, not as final proposal for concluding work. The experimental spectra shown in the following figures are averages taken from different experimental runs. The details are discussed in Section 5 . $^{62}$

\section{$4.1\left(\mathrm{H}_{2} \mathrm{O}\right)_{25}$}

In Fig. 2, we show a superposition of a typical experimental spectrum with one of our spectra simulations. The experimental data have been used without changes. The theoretical data have been scaled with a constant factor on the intensity axis, to provide a good visual match with the experimental data.

In this case, the experimental trace can be matched very well with a simulated one that is based on a single $\left(\mathrm{H}_{2} \mathrm{O}\right)_{25}$ structural isomer, which is depicted in Fig. 3. This structure does not exhibit any water molecules that could reasonably be classified as being in the interior of the cluster, i.e., it is an all-surface structure. Small water clusters always have all water molecules

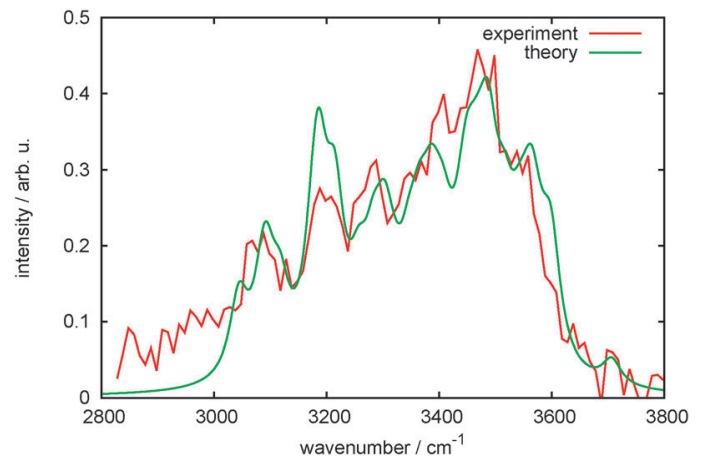

Fig. 2 Experimental (red) and simulated (green) vibrational spectra of $\left(\mathrm{H}_{2} \mathrm{O}\right)_{25}$ in the $\mathrm{OH}$-stretch region.

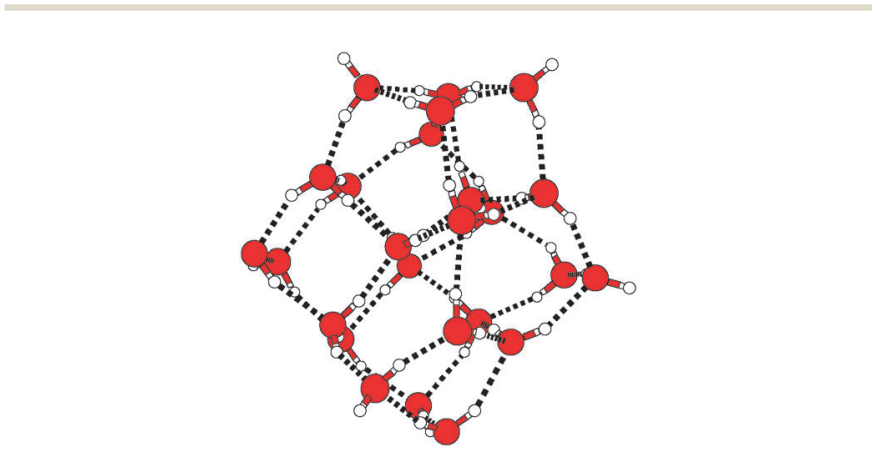

Fig. 3 The structure that dominates the $\left(\mathrm{H}_{2} \mathrm{O}\right)_{25}$ spectrum shown in Fig. 2. 
on the surface. Earlier theoretical studies ${ }^{39-41}$ have located the onset of structures with one interior molecule at $n=17$. In the same studies, however, it was also noted that for larger sizes $(n=18,19, \ldots)$ the best structures do not always have one interior water molecule. Instead, all-surface structures continue to occur among the lowest-energy local minimum-energy isomers. The present finding indicates that they may still be competitive at $n=25$. Actually, the situation seems to be even more involved than that: while among the 19 best structures we have found, the vast majority has exactly one interior molecule, there are not only two distinct all-surface structures but also one structure that features already two interior water molecules. In other words, while the transition from all-surface to one interior molecule is still not finished, the next transition to two interior molecules already begins.

Despite this interesting structural "activity" at $n=25$, the rather good match of the experimental and theoretical spectra shown in Fig. 2 leaves only minor contributions to other structures.

\section{$4.2\left(\mathrm{H}_{2} \mathrm{O}\right)_{24}$}

For $\left(\mathrm{H}_{2} \mathrm{O}\right)_{24}$, there is no single theoretical spectrum that fits the experimental one nearly as well as for $\left(\mathrm{H}_{2} \mathrm{O}\right)_{25}$. However, an equally weighted mixture of the spectra of three structures produces a rather convincing agreement (Fig. 4).

Here, from a sample of 29 best structures, about half of them (14) have all-surface structures, the other half has one

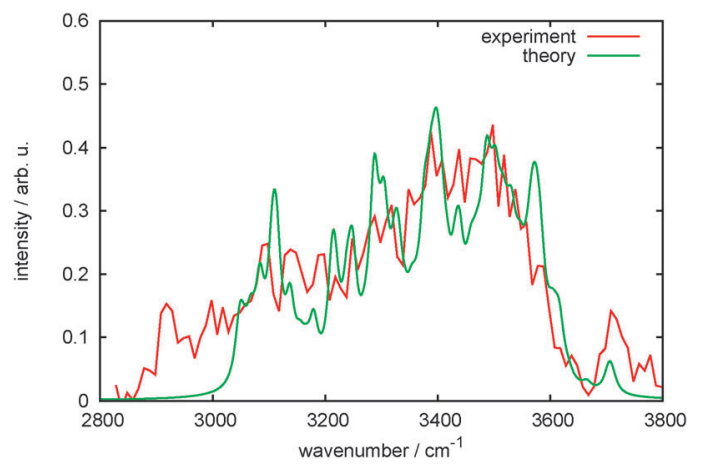

Fig. 4 Experimental (red) and simulated (green) vibrational spectra of $\left(\mathrm{H}_{2} \mathrm{O}\right)_{24}$ in the $\mathrm{OH}$-stretch region. The theoretical spectrum is generated from the three structures shown in Fig. 5.

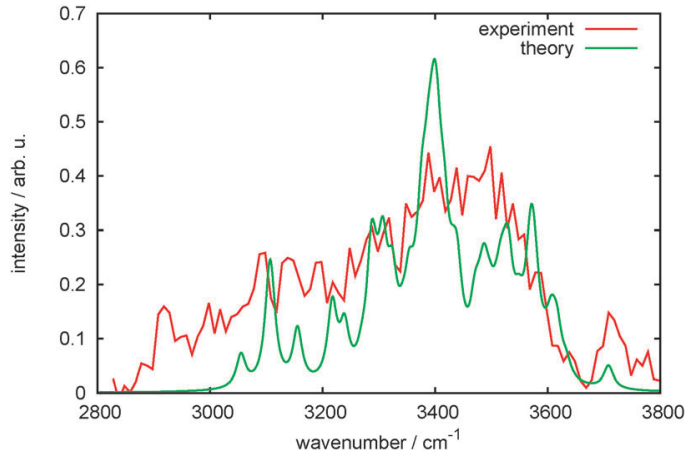

Fig. 6 Experimental (red) and simulated (green) vibrational spectra of $\left(\mathrm{H}_{2} \mathrm{O}\right)_{24}$ in the $\mathrm{OH}$-stretch region. The theoretical spectrum is generated from the two all-surface structures shown in Fig. 5.

interior water. Fig. 4 was produced with a mixture of spectra belonging to the three structures shown in Fig. 5; one of them (panel a) has one interior water molecule, the other two (panels b and c) are all-surface. Additionally, the two all-surface structure share the same water molecule positional grid and only differ in the hydrogen-bond connectivity pattern, i.e., they are monomer-orientational isomers of each other.

For comparison, in Fig. 6 we also show the simulated spectrum using only the two all-surface structures from Fig. 5, panels b and c. Arguably, the direct comparison to the experimental spectrum is somewhat worse here. However, the differences between the exp-theory-comparisons in Fig. 4 and 6 are of similar size compared to the differences between experiment and theory in both figures. Therefore, from these data, we cannot draw reliable conclusions on whether the $\left(\mathrm{H}_{2} \mathrm{O}\right)_{24}$ species observed in experiment contain interior water molecules or not.

\section{$4.3\left(\mathrm{H}_{2} \mathrm{O}\right)_{32}$}

For $\left(\mathrm{H}_{2} \mathrm{O}\right)_{32}$, the situation appears to be quite different from the cases presented above. From the top 38 unique structures generated in our global optimization, no single-structure spectrum provides a good approximation to the experimental spectrum. Likewise, superpositions of spectra from two or three structures do not work well here. In Fig. 7, we show a comparison to a superposition of seven spectra. This leads to a rather good overall spectral shape, but many deviations in the finer details are rather obvious.
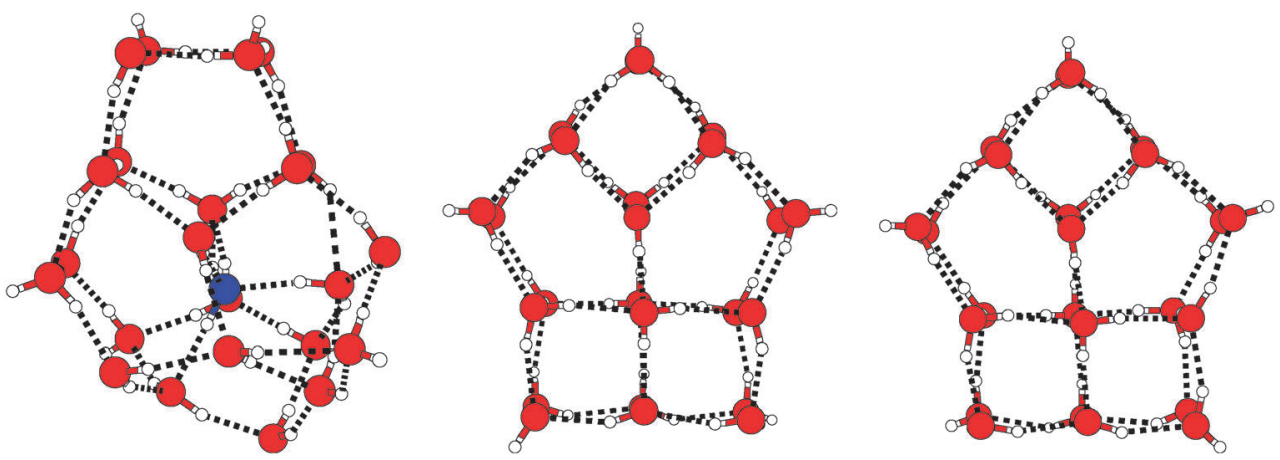

Fig. 5 The three $\left(\mathrm{H}_{2} \mathrm{O}\right)_{24}$ structures used to generate the theoretical spectrum in Fig. 4. Interior water molecules are shown with a blue oxygen atom. 


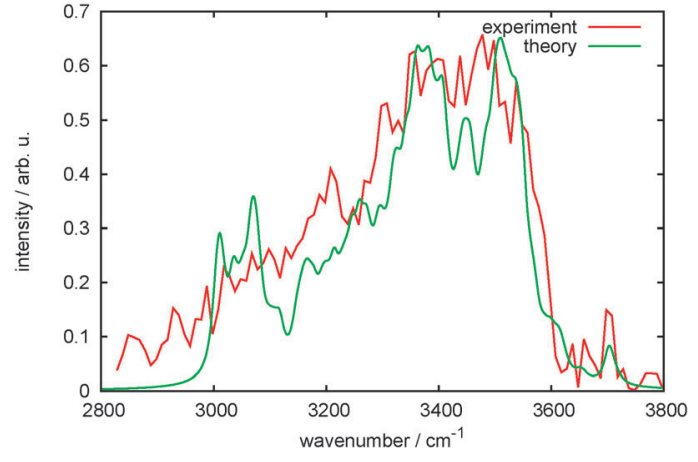

Fig. 7 Experimental (red) and simulated (green) vibrational spectra of $\left(\mathrm{H}_{2} \mathrm{O}\right)_{32}$ in the $\mathrm{OH}$-stretch region.

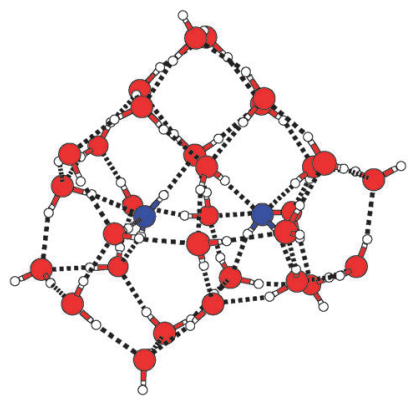

Fig. 8 One of the structures that contributes to the spectrum shown in Fig. 7. Interior water molecules are shown with a blue oxygen atom.

The actual structural variety behind this mixture of seven structures, however, is less severe than it appears. Fig. 8 gives an example for one of the structures that participate in the simulated spectrum shown in Fig. 7. This structure features two interior water molecules within a potato-shaped cage; a cuboid protrusion is located edge-down above the midpoint of an imaginary line joining the two interior water molecules. Surprisingly, all the top-38 structures just mentioned share these same general structural features (but differ in finer details). In this sense, while the assignability of one or a few structures to a given experimental spectrum may diminish for larger clusters, it may still be possible to arrive at more general conclusions regarding the observed structures.

\section{$4.4\left(\mathrm{H}_{2} \mathrm{O}\right)_{6}$}

We finish the presentation with a short discussion of the water hexamer for which the interpretation of the IR-spectra has recently been considerably improved. The clusters up to $n=10$ have unique minimum structures. While the smaller ones are characterized by rings, the larger ones are dominated by the cubic structure of the water octamer. ${ }^{8}$

The hexamer is the first 3-dimensional structure with four energetically close lying isomers. The rotational excitation spectra of the groups of Saykally ${ }^{65}$ and Pate $^{66}$ showed clear evidence for the cage structure, whereas our measurement of the vibrational $\mathrm{OH}$-stretch spectrum was interpreted as coming from the book isomer, ${ }^{63}$ because of the higher temperature in

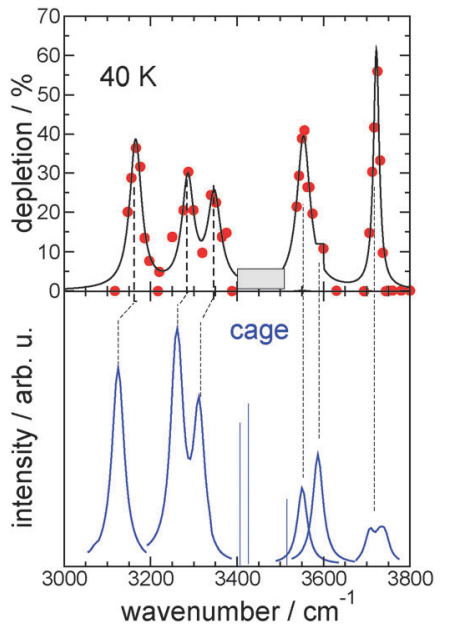

Fig. 9 Comparison of measured ${ }^{63}$ and calculated ${ }^{64} \mathrm{OH}$-stretch spectra of the water hexamer at $40 \mathrm{~K}$. The calculation is solely based on the cage isomer.

the experiment. A new very detailed and sophisticated calculation of all the hexamer isomers by the group of Skinner, however, came to the conclusion that also our measurement at $40 \mathrm{~K}$ is in full agreement with the prediction for the cage. ${ }^{64}$ The comparison is shown in Fig. 9.

We note that in this case the size selection is obtained by a completely different method compared to that described earlier, namely by deflection from a helium atomic beam. Otherwise the molecular beam conditions are similar. The pattern of the measured spectrum is completely reproduced by the calculation. The calculation is based on a mixed quantum/classical approach. The intramolecular stretches are treated quantum mechanically in a one-exciton model with anharmonic local mode frequencies including intramolecular and intermolecular coupling. The Hamiltonian is parametrized by a calculation based on a number of water molecules from an MD simulation for liquid water. The spectra at finite temperature are obtained from a replica exchange MD simulation. The systematic deviations on the small wavenumber side of the spectrum to slightly smaller values are traced back to the frequency calibration from the liquid. The measured spectrum at $60 \mathrm{~K}$ is also well reproduced by the cage isomers and a small admixture of the book isomer as is exactly predicted by theory. ${ }^{64}$ Apparently, the model which we used for the interpretation of the data in our first attempt was not good enough.

\section{Transition from amorphous to crystalline behaviour}

The introduced method to measure the $\mathrm{OH}$-stretch spectra for size selected water clusters is the ideal tool to get information on the more fundamental problem at what cluster size the crystallisation sets in. Electron diffraction measurements give crystalline structures of cubic ice for the average cluster size $\bar{n}=5000 .^{31,67}$ A transmission electron microscopy experiment shows crystalline structures for even larger sizes of some $10^{5} .^{2}$ 
In the experiment of Torchet $e t a l .{ }^{31}$ the onset of crystallinity is estimated between $n=200$ and 1000. A similar size range was covered by $\mathrm{OH}$-stretch measurements of size distributions of clusters generated in expansions and nanoparticles from collisional cooling cell experiments. ${ }^{30}$ They were in fact initiated by the pioneering calculations of Buch which were based on an anharmonic oscillator model and the intermolecular coupling between the water molecules. ${ }^{30}$ These calculations contain also the recipe to distinguish the crystalline mode from the others, the considerable shift of the maximum from $3400 \mathrm{~cm}^{-1}$ of the amorphous and the liquid peak to the $3200 \mathrm{~cm}^{-1}$ of ice.

The first experiment was carried out for a $40 \%$ water mixture in $\mathrm{He}$ at 3.9 bar and $413 \mathrm{~K}$ nozzle temperature which we denote as medium temperature for the cluster. ${ }^{29}$ The results are shown in Fig. 10 for the average sizes $\bar{n}=85$ to 475 . The smaller clusters are dominated by peaks around $3400 \mathrm{~cm}^{-1}$. For the larger clusters the main peak moves into the direction $3200 \mathrm{~cm}^{-1}$. The transition occurs around $\bar{n}=275$ with nearly equal intensity for both frequencies. At $\bar{n}=475$ we observe a clearly pronounced maximum at the position of ice. The measurements directly reflect the transition from the amorphous behaviour to the crystalline structure. At this point it is interesting to compare single experimental results with the available calculations of Buch, although they are not designed to fit the experiments.

The first comparison of a calculated $(n=123)$ spectrum with a measurement $(n=113 \pm 12)$ is presented in Fig. 11. The general trends including the maximum intensity are well reproduced by theory. The features at 3000 and $3100 \mathrm{~cm}^{-1}$ are attributed to undercoordinated single donor molecules (DAA) and those at $3500 \mathrm{~cm}^{-1}$ to double donor molecules (DDA) at

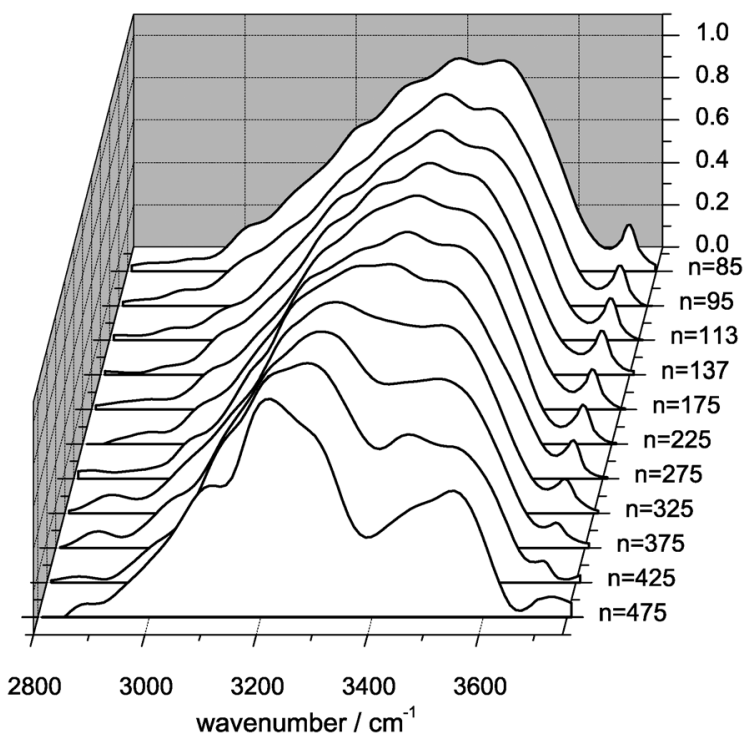

Fig. 10 IR spectra of size selected $\left(\mathrm{H}_{2} \mathrm{O}\right)_{n}$ clusters from $\bar{n}=85$ to 475 . $10(\bar{n}=85$ and 95), $25(\bar{n}=113,137)$ and $50(\bar{n}=175,225, \ldots, 475)$ cluster sizes were averaged for the plotted size slices. Expansion conditions correspond to a medium temperature (see Table 1). The IR spectra are smoothed by averaging three neighboring points $(25 \%, 50 \%, 25 \%) 5$ to 25 times. Adapted from ref. 29.

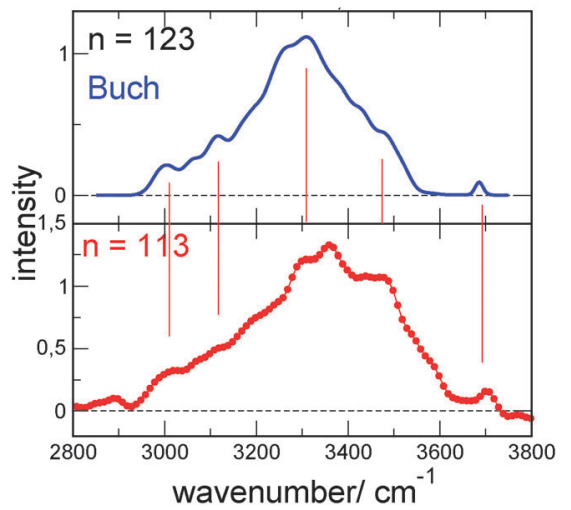

Fig. 11 Expansion conditions: 40\% water mixture in $\mathrm{Ar}$ at $1.3 \mathrm{bar}$ and $353 \mathrm{~K}$ nozzle temperature. The experimental spectrum is generated from a single IR laser scan. Data points are smoothed by three iterations of a 25, 50 and $25 \%$ weighting of neighbouring points.

the surface. The crystalline cluster $n=475$ is compared with the only calculation available in this size range for $n=600$ in Fig. 12. Again the general features are well reproduced with the maximum at $3200 \mathrm{~cm}^{-1}$ which represents the four-coordinated crystalline structures and the shoulder at $3280 \mathrm{~cm}^{-1}$ which originates from four-coordinated non-tetrahedral behaviour. The peak at $3100 \mathrm{~cm}^{-1}$ also belongs to the four-coordinated crystalline structure which can also be found in pure ice. The shoulders at 3000 and $3500 \mathrm{~cm}^{-1}$ represent three-coordinated molecules signifying that the surface of these clusters is still amorphous.

The general consistency of experiments and calculations allows us to use the predicted structures of $\mathrm{Buch}^{30}$ to give the

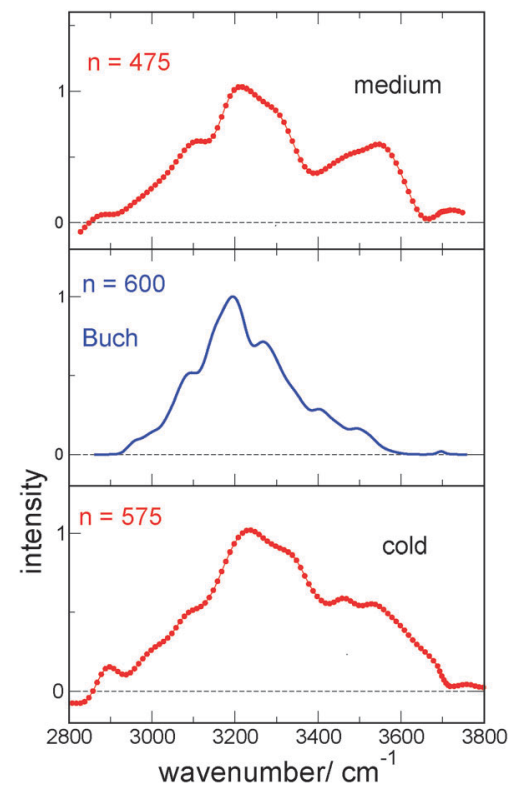

Fig. 12 Comparison of calculated IR spectra for $n=600$ with measurements for $n=475 \pm 25$ at the medium temperature and measurements for $n=575 \pm 25$ for the cold temperatures. Data points are smoothed by $15(n=575)$ and $25(n=475)$ iterations of a 25,50 and $25 \%$ weighting of neighbouring points. 


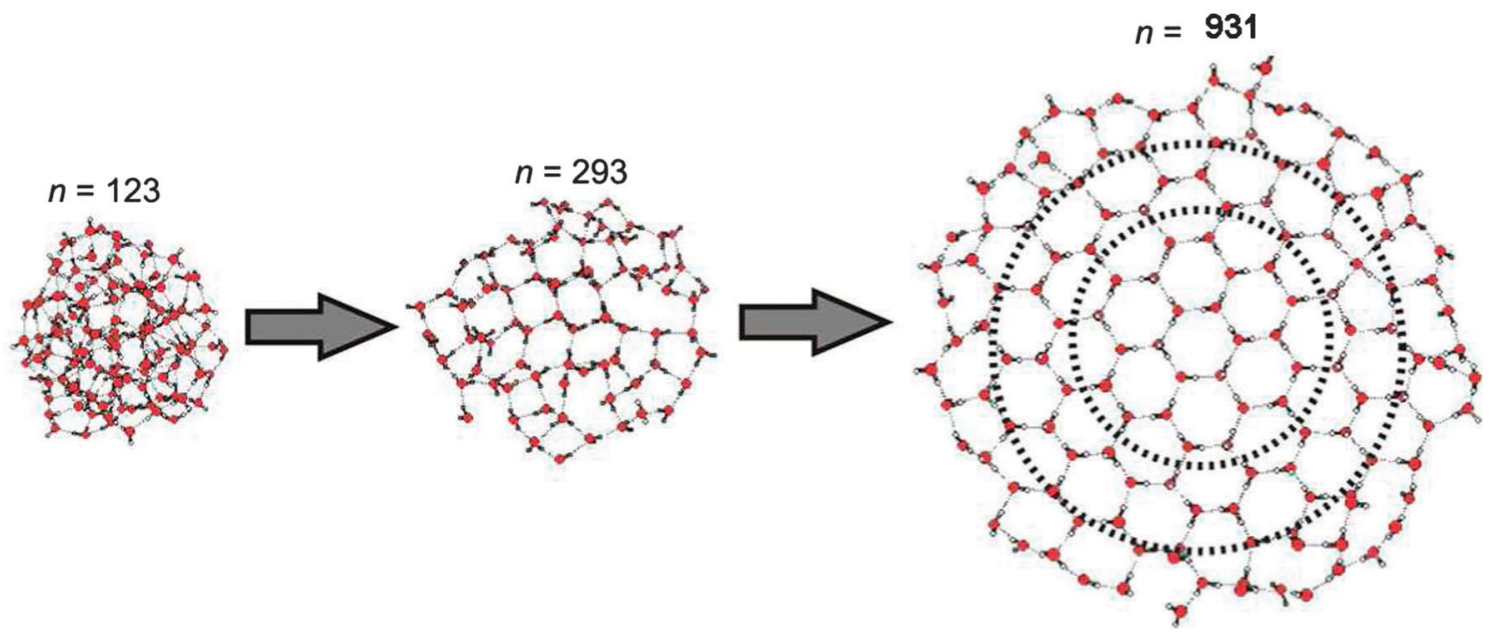

Fig. 13 Calculated transition from the amorphous to the crystalline behaviour as a function of the cluster size. The single structures are taken from ref. 30.

following picture of the crystallisation of water clusters as shown in Fig. 13. Up to a certain size the cluster structure is usually amorphous. In a specific size range the water molecules change from a disordered arrangement to a real ice crystal with hexagonal structure in the interior of the cluster. They form a ring of six molecules in a tetrahedral configuration. To begin with, this structure is still slightly deformed; however, as the cluster becomes larger, this interior grows to go over into a nicely ordered ice crystal, while the outer layers remain amorphous.

Our experiments are carried out at a fixed temperature and the effect of changing the cluster size is probed. In other experimental approaches ${ }^{68}$ and in theoretical studies, ${ }^{69}$ one usually considers one size as a function of the temperature.

Therefore it is quite interesting to repeat the experiment at different temperatures. We did that be changing the condition for the cluster beam production. The pressure was increased to 4.5 bar with quite similar conditions for the temperature (423 K) and the water contribution ( $413 \mathrm{~K}$ ). This leads to a lower cluster temperature because of the higher density of the collision partner which we denote as cold. The data are summarized in Table 1.

The cluster temperatures are based on the model which was developed for the interpretation of our data measured for $\left(\mathrm{H}_{2} \mathrm{O}\right)_{9}$ (ref. 70) and $\left(\mathrm{H}_{2} \mathrm{O}\right)_{6}$ (ref. 63) (see Fig. 9). The result of the curve with the most pronounced peak at $3200 \mathrm{~cm}^{-1}$ is also displayed in Fig. 12. It looks very similar to the previously measured curve and follows closely the calculated curve for $n=600$, but it belongs to the size $\bar{n}=575$. In this experiment, however, the IR spectrum at $\bar{n}=475$ peaks around $3300 \mathrm{~cm}^{-1}$ in the transition region. This definitely means that the crystallisation occurs at higher cluster sizes when the temperature is lowered.

Table 1 Production conditions

\begin{tabular}{lllllc}
\hline Beam & Gas & $p /$ bar & $T / \mathrm{K}$ & $p\left(\mathrm{H}_{2} \mathrm{O}\right)$ & $T(\mathrm{clu}) / \mathrm{K}$ \\
\hline Cold & $\mathrm{He}$ & 4.5 & 423 & 3.58 & 50 \\
Medium & $\mathrm{He}$ & 3.9 & 413 & 2.67 & 70 \\
Warm & $\mathrm{Ne}$ & 2.3 & 433 & 1.00 & 100
\end{tabular}

More molecules are necessary to form the hexagonal structure. In order to investigate this effect in more detail, we have plotted the IR-spectra as a function of the cluster size in a continuous contour plot of the intensity for both the medium and the cold temperature in Fig. 14 and 15. In the first experiment at medium temperature we observe for the smaller sizes a pronounced peak at $3400 \mathrm{~cm}^{-1}$ indicating the amorphous structure. Then suddenly a jump to smaller wavenumbers occurs around $n=275$ followed by an additional one at $n=425$ leading to the peak close $3200 \mathrm{~cm}^{-1}$ at $n=475$. For the cold arrangement the first jump occurs at much larger values around $n=425$ and ends up in a more continuous way at $n=575$.

To check if the effect also works in the other direction, we generated a water cluster beam by expanding a mixture of water at $372 \mathrm{~K}$ in Ne at 2.3 bar and 383 to $433 \mathrm{~K}$. This should give a higher temperature compared to the first experiment with a medium temperature which we denote as warm. The cluster temperature for this case presented in Table 1 has been calculated by Gimelshein ${ }^{71}$ based on simulation methods of the flow and the

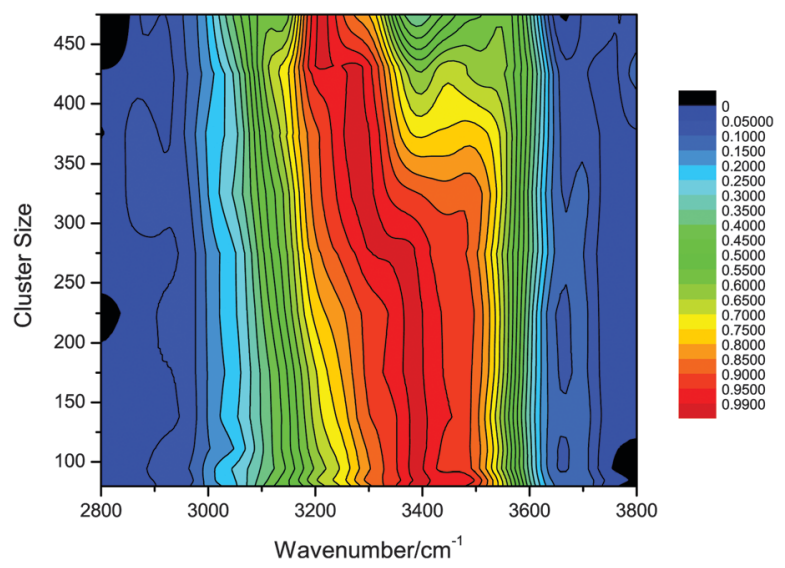

Fig. 14 Continuous contour map of the IR-spectra taken at medium temperature. Note the two transitions in the maximum intensity at $n=275$ and $n=425$. 


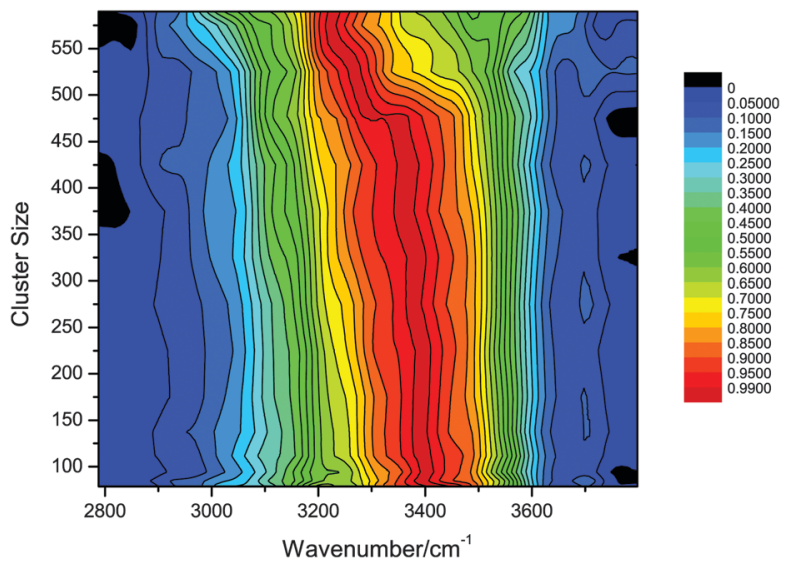

Fig. 15 Continuous contour map of the IR-spectra taken at cold temperature. Note the two transitions in the maximum intensity at $n=425$ and $n=575$.

condensation in and outside conical nozzles. ${ }^{72,73}$ The simulation gives the final cluster temperature after the molecular beam has been established. However, we note that the heat of crystallization may change the cluster temperatures. This effect will be inspected in detail in future work. In the case of the warm cluster we could not measure up to similar large clusters as in the first two other examples, because large clusters with $n>300$ are not formed in detectable abundances under these experimental conditions. However, we found clear indications that the crystallization process has definitely started at lower cluster sizes. The onset of crystallization is indicated by the shift of the absorption maximum and by specific absorption patterns, which are absent in amorphous clusters. These findings are illustrated in Fig. 16. The lower panel shows IR spectra of amorphous clusters, averaged for the cluster sizes $n=100$ to 125 . Both traces belong to an averaged spectrum of three IR laser scans under either medium (red trace) or warm (black trace) conditions. The direct comparison demonstrates aside from noise and possibly some subtle structure complete agreement and thus the robustness of the $\mathrm{OH}$ stretch spectra in this size range with regard to cluster temperature (see also Fig. 11 for an experiment with an Ar seeded expansion which corresponds to a cold cluster temperature and which also agrees with the other two experiments).

The situation changes dramatically when we look at the IR spectra for larger cluster sizes of the same series of experiments (red and black trace in the upper panel). Clearly, the position of the maximum absorption peak has shifted to the lower frequencies of the crystalline arrangement. This shift is very sensitive to the nozzle temperature. Increasing the temperature decreases the critical cluster size for the onset of crystallization. We note again that the size slices for the warm and the medium case belong to the same set of experiments as shown in the lower panel for the amorphous case. To be more specific, the spectra are measured simultaneously with identical experimental conditions for each single IR and UV laser shot. The experimental trace for the warm expansion belongs to an average cluster size of $\bar{n}=225$ (cluster sizes in three experiments: $n=140$ to 275). The comparison with the result of the crystalline cluster for $\bar{n}=475$ at medium temperatures exhibits remarkable similarities but also

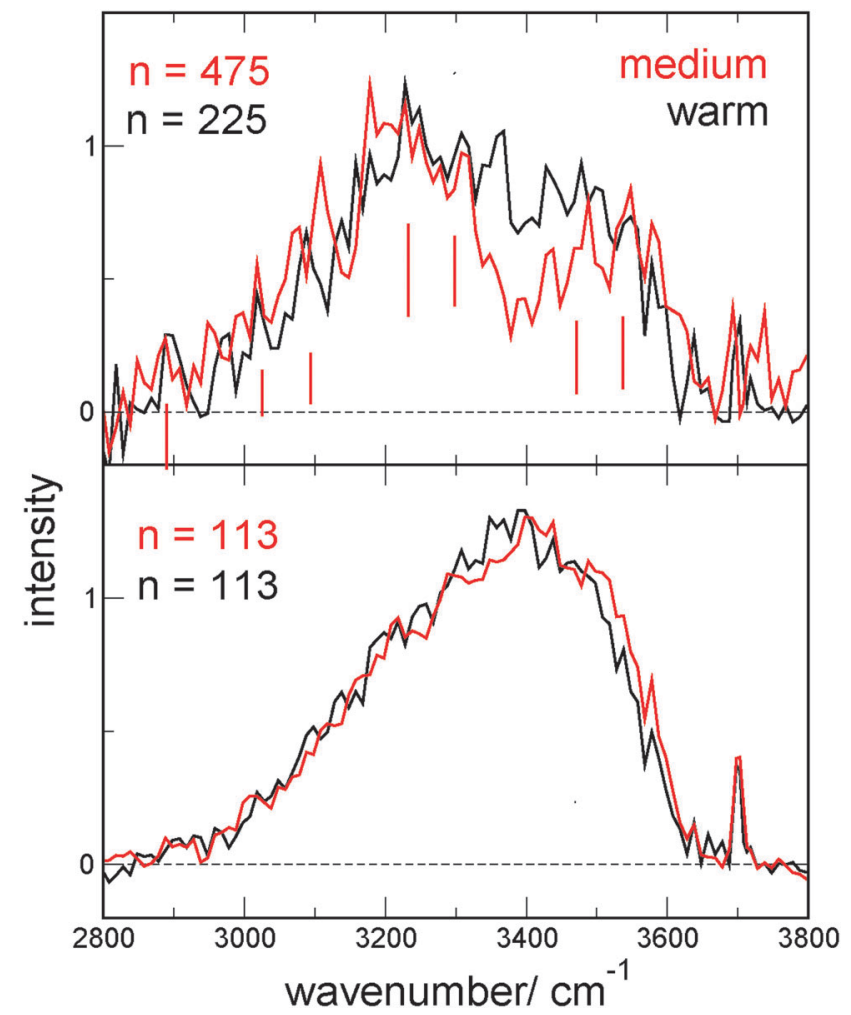

Fig. 16 Comparison of amorphous and crystalline structures for warm cluster temperature (black) with those obtained for medium temperatures (red) from Fig. 11 and 12.

distinct differences. The spectrum for $\bar{n}=475$ in Fig. 16 is also an average of three experiments, but different from the presentation in Fig. 12 and in ref. 29 it is shown here without any smoothening. We opted for presenting smoothed spectra in the previous work because we had not enough evidence that the spiky features are robust. However, the comparison in the upper panel of Fig. 16 clearly shows that both spectra are very structured with good agreement in the range around 3200 to $3300 \mathrm{~cm}^{-1}$. The largest differences occur in the frequency range between $3300 \mathrm{~cm}^{-1}$ and $3500 \mathrm{~cm}^{-1}$. The $n=475$ spectrum has a clear two peak structure with the smaller peak around $3500 \mathrm{~cm}^{-1}$ in the range of three-coordinated DDA molecules, while the $n=225$ spectrum shows higher intensities in the range of $3400 \mathrm{~cm}^{-1}$, the domain of amorphous behaviour.

A further important observation with regard to the onset of crystallization is the appearance of distinct and reproducible absorption features around $2900 \mathrm{~cm}^{-1}$ and $3000 \mathrm{~cm}^{-1}$ in the region of DAA oscillators (2800 to $3150 \mathrm{~cm}^{-1}$ ). The same features are present in the smoothed IR spectra of the colder, He seeded expansion (see Fig. 12). The feature around $2900 \mathrm{~cm}^{-1}$ has been neither observed in large amorphous clusters in our size selective experiments nor in the IR spectra of crystalline ice particles generated from the complete size distribution in collisional cooling cell experiments. ${ }^{30,74}$ First we note that our IR action spectrum does not show the true absorption behaviour. We further note that the average cluster sizes in our experiments are much lower than the largest size slices in which the crystalline 
clusters appear. In terms of both cluster masses and cluster numbers the fraction of clusters with largely crystalline structures may represent less than ten percent of the total cluster mass and number. Interestingly, this effect is most pronounced for the experiment in which the largest clusters are produced, because also the crystallization starts at larger cluster sizes (see Fig. 15). This observation shows that cluster generation conditions and the spectral properties of clusters can be tightly coupled, which complicates the interpretation of experiments in which the spectral features represent the mean cluster properties of typically broad size distributions. In fully size selective experiments the connection to the mean cluster properties is broken.

The $\mathrm{OH}$ stretch spectra of larger water clusters, generated with the newly available size selective techniques for both neutral ${ }^{22}$ and ionic ${ }^{11,75}$ clusters have in common that they reveal spectral structure. Interestingly, both the envelope and the subtle spectral features of $\mathrm{OH}$ stretch spectra of still amorphous clusters for sizes around $n=225$ in our experiment (medium temperature) are almost identical to those of cationic $\mathrm{Na}^{+}\left(\mathrm{H}_{2} \mathrm{O}\right)_{n}$ clusters for $n=250 . .^{29,75}$ The IR spectra were generated exploiting very different action effects (signal enhancement as explained above in our experiment, ensemble infrared photodissociation in the work of Williams and co-workers). The experiments of the Williams group revealed the clear influence of charge on the peak position of the free $\mathrm{OH}$ stretch mode and rather subtle features in the spectral region of bonded $\mathrm{OH}$ oscillators. In the case of small crystalline water clusters the new experiments reveal that the ice nucleation is a function of temperature and definitely occurs at smaller sizes for higher temperatures. A surprise is that the small crystalline clusters show a distinct, spiky IR absorption pattern, indicating that these clusters have a characteristic surface, which is different from both amorphous clusters and large crystalline nanoparticles. ${ }^{74}$ The feature around $2900 \mathrm{~cm}^{-1}$ belongs probably to a specific DAA motif. The origin of the feature around $3100 \mathrm{~cm}^{-1}$ and the spiky character in general may be complex and could involve DDAA oscillators and couplings, which are related to proton disorder or, perhaps more likely, to an orientationally ordered surface and interior of the clusters generated in this type of experiment. ${ }^{76}$

To find reasons for this interesting behaviour is a challenge for the theory. Nearly all of the most recent calculations are not exactly designed for the present problem. They mainly treat the crystallisation from the liquid to the solid transition at different temperatures. ${ }^{69,77,78}$ Aside from using Molecular Dynamics (MD) simulations based on the monatomic water model which represents each water molecule by a single particle with a realistic three-body interaction, ${ }^{79}$ they partly integrate elements of the classical nucleation theory which is not valid for our type of experiment. In supersonic expansions clusters are produced from the gas phase and the final cluster temperature is reached after a period of cooling by collisions with the seed gas. Hence the temperature histories of the clusters (e.g. the time period during which they are supercooled liquid, or the release of latent heat) are probably also important for the interpretation of this type of experiment. The work which comes closest to our results is that of Johnston and Molinero. ${ }^{77}$ They found freezing temperatures of $155 \mathrm{~K}$ for $n=159$ and $179 \mathrm{~K}$ for $n=417$ well above the temperatures of our experiment which explains that we do not observe any indication of the liquid. Nevertheless they observe in both cases structures with a crystallized core and surrounding liquid-like molecules similar to that found in the calculations of Buch, see Fig. $13 .{ }^{30}$ In addition, we definitely observe the onset of crystalline behaviour below $n=200$ at the warm temperature which we attribute to about $100 \mathrm{~K}$. This is not far away from the results obtained by Molinero and coworkers. ${ }^{77}$

\section{Summary}

The doping with $\mathrm{Na}$ atoms and the subsequent fragmentation free photoionization proved to be an effective method for generating size selected water clusters. In combination with IR-photon excitation the $\mathrm{OH}$-stretch spectra of water clusters up to $n=600$ are obtained in a very efficient way. We present here the application in two different fields, the determination of the cluster structure and the onset of crystallinity.

As for the structure assignment, this work extends the theoretical spectroscopy of water clusters significantly beyond the previously studied size range of $n=6-12$. The energy landscapes of those smaller clusters feature only comparatively very few structural isomers and large energy differences between them. As discussed in Section 3.3, this is very different already in the size region $n \geq 20$, turning the correct modelling of water clusters of these sizes into a challenge. Nevertheless, this work demonstrates in Sections 4.1-4.3 that direct comparisons of IR spectra between theory and experiments are possible and beneficial. They allow us to draw first tentative conclusions regarding qualitative structural properties of water clusters in this size range and under the experimental conditions presented here. Even if the number of structures contributing to these spectra cannot be narrowed down to just a few in all cases, this work presents first steps toward a general simulation protocol of multistructural theoretical cluster spectroscopy.

The prospects to explain vibrational water cluster spectra from theory also in the more interesting but still larger size regime of the onset of crystallinity seem bleak at first glance. One possible solution is to relax comparison standards from matching single peaks to only matching spectral envelopes qualitatively. As shown in Section 5, this approach offers useful insights. To go into more detail we will require substantial further progress in simulation approaches.

The first measurement of the onset of crystallisation of water clusters on a complete size resolved basis sets the endpoint of a long series of theoretical predictions and the speculative interpretation of experiments with size distributions, see Section 5. The surprising result of our investigations was the dependence of this transition on the cluster temperature. For expansion conditions leading to cold clusters we measured a completed transition at $\bar{n}=575$. The IR-spectrum resembles very much the calculation of Buch ${ }^{30}$ as shown in Fig. 12. All the peaks and shoulders between $3100 \mathrm{~cm}^{-1}$ and $3300 \mathrm{~cm}^{-1}$ that represent the four-coordinated crystalline behaviour as well as 
the ones at $3000 \mathrm{~cm}^{-1}$ and $3500 \mathrm{~cm}^{-1}$ which stand for threecoordinated amorphous surface behaviour are present. For clusters at medium temperatures the transition is completed at slightly smaller sizes of $\bar{n}=475$. The IR-spectrum has a similar shape with the difference that around $3500 \mathrm{~cm}^{-1}$ a peak structure evolves, see Fig. 12 and 16. At a still higher temperature, the warm case, the transition is observed at the average cluster size of $\bar{n}=225$. Although a number of lines in the IR-spectra taken at the medium and warm temperature are quite similar, there is a difference with regard to the appreciable intensity around $3400 \mathrm{~cm}^{-1}$, the original peak position of the amorphous behaviour. Apparently, the crystallisation is almost completed within this size range, but a larger part of the cluster is still amorphous compared to $\bar{n}=475$.

We note that these are the first experiments in this direction so that we expect many more results in the future which will help to clarify the situation. In addition, we might think of extending the experiments to still higher temperatures so that also the liquid to crystalline transition is investigated including the coexistence of solid and liquid parts of the cluster. ${ }^{80}$

\section{Acknowledgements}

TZ thanks Prof. Martin Suhm for his ongoing support and acknowledges funding by the Deutsche Forschungsgemeinschaft (grants ZE 890 1-1, 2 and GRK 782). UB and TZ thank Dr Richard Forck, Christoph Dierking and Florian Zurheide for their contributions to the experimental part of this work and the tedious evaluation and processing of the experimental data. JMD and $\mathrm{BH}$ would like to thank Insa Stamer for patiently performing many global optimization runs with ogolem at the TTM3F-level, in her BSc thesis work.

\section{References}

1 E. M. Knipping, M. J. Lakin, K. L. Foster, P. Jungwirth, D. J. Tobias, R. B. Gerber, D. Dabdub and B. J. FinlaysonPitts, Science, 2000, 288, 301.

2 L. Delzeit and D. Blake, J. Geophys. Res.: Planets, 2001, 106, 33371.

3 M. Kulmala, Science, 2003, 302, 1000.

4 Q. Shi, S. D. Belair, J. S. Francisco and S. Kais, Proc. Natl. Acad. Sci. U. S. A., 2003, 100, 9686.

5 R. Zhang, A. Khalizov, L. Wang, M. Hu and W. Xu, Chem. Rev., 2012, 112, 1957.

6 C. P. Lawrence and J. L. Skinner, J. Chem. Phys., 2003, 118, 264.

7 T. Schäfer, J. Lindner, P. Vöhringer and D. Schwarzer, J. Chem. Phys., 2009, 130, 224502.

8 U. Buck and F. Huisken, Chem. Rev., 2000, 100, 3863.

9 M. A. Suhm, Adv. Chem. Phys., 2009, 142, 1.

10 M. A. Suhm and F. Kollipost, Phys. Chem. Chem. Phys., 2013, 15, 10702.

11 A. Fujii and K. Mizuse, Int. Rev. Phys. Chem., 2013, 32, 266.

12 K. E. Otto, Z. Xue, P. Zielke and M. A. Suhm, Phys. Chem. Chem. Phys., 2014, DOI: 10.1039/c3cp54272f.
13 U. Buck and H. Meyer, Phys. Rev. Lett., 1984, 52, 109.

14 U. Buck, J. Phys. Chem., 1994, 98, 5190.

15 U. Buck, I. Ettischer, M. Melzer, V. Buch and J. Sadlej, Phys. Rev. Lett., 1998, 80, 2578.

16 K. Liu, J. D. Cruzan and R. J. Saykally, Science, 1996, 271, 929.

17 E. G. Diken, W. H. Robertson and M. A. Johnson, J. Phys. Chem. A, 2004, 108, 64.

18 C. J. Gruenloh, J. R. Carney, C. A. Arrington, T. S. Zwier, S. Y. Fredericks and K. D. Jordan, Science, 1997, 276, 1678.

19 T. Watanabe, T. Ebata, S. Tanabe and N. Mikami, J. Chem. Phys., 1996, 105, 408.

20 W. Roth, M. Schmitt, C. Jacoby, D. Spangenberg, C. Janzen and K. Kleinermanns, Chem. Phys., 1998, 239, 1.

21 K. Mizuse, T. Hamashima and J. Fujii, J. Phys. Chem. A, 2009, 113, 12134.

22 T. Hamashima, K. Mizuse and A. Fujii, J. Phys. Chem. A, 2011, 115, 620.

23 S. Schütte and U. Buck, Int. J. Mass Spectrom., 2002, 220, 1887.

24 C. Bobbert, S. Schütte, C. Steinbach and U. Buck, Eur. Phys. J. D, 2002, 19, 183.

25 C. Steinbach and U. Buck, J. Phys. Chem. A, 2006, 110, 3128. 26 U. Buck, I. Dauster, B. Gao and Z. F. Liu, J. Phys. Chem. A, 2007, 111, 12355.

27 R. M. Forck, C. C. Pradzynski, S. Wolff, M. Ončák, P. Slavíček and T. Zeuch, Phys. Chem. Chem. Phys., 2012, 14, 3004.

28 R. M. Forck, J. M. Dieterich, C. C. Pradzynski, A. L. Huchting, R. A. Mata and T. Zeuch, Phys. Chem. Chem. Phys., 2012, 14, 9054 .

29 C. C. Pradzynski, R. M. Forck, T. Zeuch, P. Slavíček and U. Buck, Science, 2012, 337, 1529.

30 V. Buch, S. Bauerecker, J. P. Devlin, U. Buck and J. K. Kazimirski, Int. Rev. Phys. Chem., 2004, 23, 375.

31 G. Torchet, P. Schwartz, J. Farge, M. F. de Feraudy and B. Raoult, J. Chem. Phys., 1983, 79, 6196.

32 I. V. Hertel, C. Hüglin, C. Nitsch and C. P. Schulz, Phys. Rev. Lett., 1991, 67, 1767.

33 T. Zeuch and U. Buck, Chem. Phys. Lett., 2013, 579, 1.

34 R. M. Forck, I. Dauster, Y. Schieweck, T. Zeuch, U. Buck, M. Ončák and P. Slavíček, J. Chem. Phys., 2010, 132, 221102.

35 L. T. Wille and J. Vennik, J. Phys. A: Math. Gen., 1985, 18, L419.

36 B. Hartke, Z. Phys. Chem., 2000, 214, 1251.

37 D. J. Wales and M. P. Hodges, Chem. Phys. Lett., 1998, 286, 65.

38 C. J. Burnham and S. S. Xantheas, J. Chem. Phys., 2002, 116, 5115.

39 B. Hartke, Phys. Chem. Chem. Phys., 2003, 5, 275.

40 B. Bandow and B. Hartke, J. Phys. Chem. A, 2006, 110, 5809.

41 A. Lagutschenkov, G. S. Fanourgakis, G. Niedner-Schatteburg and S. S. Xantheas, J. Chem. Phys., 2005, 122, 194310.

42 S. Kazachenko and A. J. Thakkar, J. Chem. Phys., 2013, 138, 194302.

43 B. Hartke, Angew. Chem., Int. Ed., 2002, 41, 1468.

44 B. Hartke, WIREs Comput. Mol. Sci., 2011, 1, 879. 
45 J. M. Dieterich and B. Hartke, Mol. Phys., 2010, 108, 279; also see http://www.ogolem.org/.

46 D. E. Goldberg, Genetic Algorithms in Search, Optimization and Machine Learning, Addison-Wesley, Reading, 1989.

47 J. M. Dieterich and B. Hartke, J. Comput. Chem., 2011, 32, 1377.

48 B. Hartke, J. Comput. Chem., 1999, 20, 1752.

49 D. H. Ackley, A Connectionist Machine for Genetic Hill Climbing, Kluwer Academic Publishers, Dordrecht, 1987.

50 J. M. Dieterich and B. Hartke, Appl. Math., 2012, 3, 1552.

51 R. Czerminski and R. Elber, J. Chem. Phys., 1990, 92, 5580.

52 J. C. Schön, H. Putz and M. Jansen, J. Phys.: Condens. Matter, 1996, 8, 143.

53 J. P. K. Doye, M. A. Miller and D. J. Wales, J. Chem. Phys., 1999, 111, 8417.

54 P. Sibani, J. Schön, P. Salamon and J. O. Andersson, Europhys. Lett., 1993, 22, 479.

55 R. F. P. Salamon and P. Sibani, Facts, Conjectures, and Improvements for Simulated Annealing, Monographs on Mathematical Modeling and Computation, SIAM, 2002.

56 G. S. Fanourgakis and S. S. Xantheas, J. Chem. Phys., 2008, 128, 074506.

57 J. Sadlej, V. Buch, J. K. Kazimirski and U. Buck, J. Phys. Chem. A, 1999, 103, 4933.

58 J. Kim and K. S. Kim, J. Chem. Phys., 1998, 109, 5886.

59 A. Lenz and L. Ojamäe, Chem. Phys. Lett., 2006, 418, 361.

60 A. Lenz and L. Ojamäe, J. Chem. Phys., 2009, 131, 134302.

61 A. Lenz and L. Ojamäe, THEOCHEM, 2010, 944, 163.

62 The conditions for the different runs are given in Table 1 for Ne seeded and in the captions of Fig. 11 for Ar seeded expansions. The spectra for $n=25$ and $n=32$ are averaged over 8 experiments ( 4 in $\mathrm{Ne}+4$ in $\mathrm{Ar}$ expansions). The spectra for $n=24$ are averaged over 4 experiments in $\mathrm{Ne}$ expansions. Here the Ar expansions can alter the isomer distribution. The detailed inspection of this effect is work in progress. The IR laser frequency is calibrated by comparison with FTIR spectra of polystyrol and polyethylene foil. The spectra are not corrected for IR laser pulse energies which has only a small effect on the spectral shape.

63 C. Steinbach, P. Andersson, M. Melzer, J. K. Kazimirski, U. Buck and V. Buch, Phys. Chem. Chem. Phys., 2004, 6, 3320. 64 C. J. Tainter and J. L. Skinner, J. Chem. Phys., 2012, 137, 104304. 65 K. Liu, M. G. Brown and R. J. Saykally, J. Phys. Chem., 1997, $101,8995$.

66 C. Perez, M. T. Muckle, D. P. Zaleski, N. A. Seifert, B. Temelso, G. C. Shields, Z. Kisiel and B. H. Pate, Science, 2012, 336, 897.

67 J. Huang and L. S. Bartell, J. Phys. Chem., 1995, 99, 3924.

68 C. Hock, M. Schmidt, R. Kuhnen, C. Bartels, L. Ma, H. Haberland and B. v. Issendorff, Phys. Rev. Lett., 2009, 103, 073401.

69 E. B. Moore and V. Molinero, Nature, 2011, 479, 506.

70 J. Brudermann, U. Buck and V. Buch, J. Phys. Chem., 2002, 106, 453.

71 N. Gimelshein and S. Gimelshein, Unpublished results, 2012.

72 R. Jansen, I. Wysong, S. Gimelshein, M. Zeifman and U. Buck, J. Chem. Phys., 2010, 132, 244105.

73 R. Jansen, N. Gimelshein, S. Gimelshein and I. Wysong, J. Chem. Phys., 2011, 134, 244105.

74 C. Medcraft, D. McNaughton, C. D. Thompson, D. R. T. Appadoo, S. Bauerecker and E. G. Robertson, Phys. Chem. Chem. Phys., 2013, 15, 3630.

75 J. T. O'Brien and E. R. Williams, J. Am. Chem. Soc., 2012, 134, 10228.

76 V. Buch, H. Groenzin, I. Li, M. J. Shultz and E. Tosatti, Proc. Natl. Acad. Sci. U. S. A., 2008, 105, 5969.

77 J. C. Johnston and V. Molinero, J. Am. Chem. Soc., 2012, 134, 6650.

78 T. Li, D. Donaldo and G. Galli, Nat. Commun., 2013, 4, 1.

79 V. Molinero and E. B. Moore, J. Phys. Chem. B, 2009, 113, 4008.

80 D. J. Wales and R. S. Berry, J. Chem. Phys., 1990, 92, 4473. 